\title{
Proton Therapy
}

for HPV-Associated

Oropharyngeal Cancers

of the Head and Neck:

a De-Intensification Strategy

Nicolette Taku, MD MPhil MPH

Li Wang, MD PhD 2

Adam S. Garden, $M D^{1}$

David I. Rosenthal, $M D^{1}$

G. Brandon Gunn, $M D^{1}$

William H. Morrison, $M D^{1}$

C. David Fuller, MD $P h D^{1}$

Jack Phan, MD PhD ${ }^{1}$

Jay $P$. Reddy, $M D^{1}$

Amy C. Moreno, $M D^{1}$

Michael T. Spiotto, MD PhD ${ }^{1}$

Gregory Chronowski, MD ${ }^{1}$

Shalin J. Shah, $M D^{1}$

Lauren L. Mayo, MD ${ }^{1}$

Neil D. Gross, $M D^{3}$

Renata Ferrarotto, $M D^{4}$

X. Ronald Zhu, PhD ${ }^{5}$

Xiaodong Zhang, $P h D^{5}$

Steven J. Frank, $M D^{1 \text {, }}$

\section{Address}

${ }^{*}, 1$ Department of Radiation Oncology, The University of Texas MD Anderson Cancer

Center, 1515 Holcombe Boulevard, Houston, TX, 77030, USA

Email: sjfrank@mdanderson.org

2Department of Experimental Radiation Oncology, The University of Texas MD Anderson Cancer Center, Houston, TX, USA

${ }^{3}$ Department of Head and Neck Surgery, The University of Texas MD Anderson

Cancer Center, Houston, TX, USA 


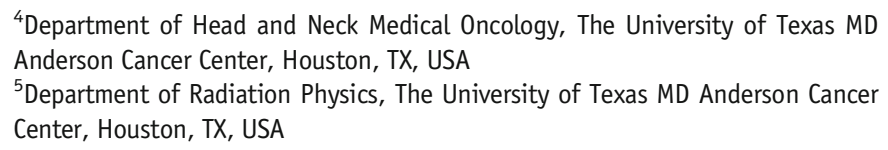

Published online: 4 June 2021

(C) The Author(s) 2021. This article is an open access publication

This article is part of the Topical Collection on Head and Neck Cancer

Keywords Head and neck · Proton therapy · De-intensification • Oropharyngeal cancer · Clinical trials · De-escalation

\section{Opinion statement}

The rise in the incidence of human papillomavirus (HPV)-associated oropharyngeal squamous cell carcinoma (OPC), the relatively young age at which it is diagnosed, and its favorable prognosis necessitate the use of treatment techniques that reduce the likelihood of side effects during and after curative treatment. Intensity-modulated proton therapy (IMPT) is a form of radiotherapy that de-intensifies treatment through dose de-escalation to normal tissues without compromising dose to the primary tumor and involved, regional lymph nodes. Preclinical studies have demonstrated that HPV-positive squamous cell carcinoma is more sensitive to proton radiation than is HPV-negative squamous cell carcinoma. Retrospective studies comparing intensity-modulated photon (X-ray) radiotherapy to IMPT for OPC suggest comparable rates of disease control and lower rates of pain, xerostomia, dysphagia, dysgeusia, gastrostomy tube dependence, and osteoradionecrosis with IMPT-all of which meaningfully affect the quality of life of patients treated for HPV-associated OPC. Two phase III trials currently underway - the "Randomized Trial of IMPT versus IMRT for the Treatment of Oropharyngeal Cancer of the Head and Neck" and the "TOxicity Reduction using Proton bEam therapy for Oropharyngeal cancer (TORPEd0)" trial—are expected to provide prospective, level I evidence regarding the effectiveness of IMPT for such patients.

\section{Introduction}

With a projected incidence of 65,000 cases in 2020 , cancers of the head and neck (HNC) constitute approximately $3 \%$ of malignancies in the USA [1]. External beam radiotherapy (EBRT) is important in the management of $\mathrm{HNC}$, with $75 \%$ of HNC patients undergoing EBRT as either primary or postoperative therapy [2]. Although the overall incidence of HNC has declined in recent decades, the incidence of oropharyngeal cancer (OPC) associated with human papillomavirus (HPV) infection has increased, with 70-90\% of newly diagnosed OPC cases showing molecular findings consistent with HPV positivity [3]. Patients with HPV-associated OPC are more likely to be diagnosed in the 4th or 5th decade of life and have a more favorable prognosis than patients with smoking-associated (HPV-negative) cancers-underscoring the need for precise, conformal radiotherapy that minimizes the likelihood of longterm, treatment-related side effects. 


\section{HPV-associated oropharyngeal cancer}

Approximately 15,800 cases of HPV-associated OPC are diagnosed annually in the USA [4]. Transmitted via orogenital contact, HPV infects the basal epithelial layer of the oropharyngeal mucosa and integrates itself into the host genome. The HPV oncoproteins E6 and E7 bind and degrade the host p53 and Rb tumor suppressor proteins, respectively, inhibit cell death pathways, and promote cellular proliferation [5]. Infection with high-risk HPV subtypes, of which HPV16 is the most common, can result in cancers of the tonsils and base of the tongue. HPV-associated cancers of the other oropharyngeal subsites (uvula, soft palate, and posterior pharyngeal wall) are less common.

Some early-stage HPV-associated OPC, particularly in "never-smokers" or smokers with a less than 10-pack-year history, can be managed with surgery or EBRT $[6,7]$. However, because disease often presents with extensive lymphadenopathy or radiographic evidence of extranodal extension, definitive chemoradiotherapy or combined treatment with surgery followed by adjuvant radiotherapy (with or without chemotherapy) is the mainstay of treatment [8]. Consensus guidelines recommend radiation doses of 66-70 Gy for definitive treatment and 60-66 Gy for postoperative radiation [9].

For patients receiving chemoradiotherapy, cisplatin is the agent of choice over other systemic agents, including cetuximab. Trial 1016 from the Radiotherapy Oncology Group (RTOG) was a treatment de-intensification study with a non-inferiority design that compared EBRT given with either the alkylating chemotherapy agent cisplatin or the epidermal growth factor receptor (EGFR)neutralizing antibody cetuximab, with the hypothesis that concurrent cetuximab would yield high rates of patient survival and lower rates of treatment-related toxicity [10]. At a median interval of 4.5 years of follow-up, clinical outcomes for cetuximab concurrent with radiotherapy failed to meet the predetermined non-inferiority criterion, in that the 5 -year overall survival rate for patients given concurrent cetuximab $(77.9 \%$; 95\% confidence interval (CI) 73.4-82.5) was lower than that achieved with concurrent cisplatin (84.6\%; 95\% CI 80.6-88.6). Progression-free survival and locoregional control rates were also worse with cetuximab. However, rates of moderate to severe acute and late side effects were comparable between the two treatment groups [10]. Similar outcomes were observed in the De-ESCALaTE HPV study, a phase III trial comparing chemoradiotherapy with cisplatin versus cetuximab for low-risk (i.e., never-smokers or smokers with a less than 10-pack-year history) HPVassociated OPC, and ARTSCAN III, a randomized phase III trial comparing chemoradiotherapy with cisplatin versus cetuximab for locoregionally advanced squamous cell carcinoma of the head and neck [11, 12].

\section{Radiotherapy treatment side effects}

As is true for chemotherapy, considerable effort has been dedicated to minimizing the side effects of EBRT experienced during and after treatment for OPC. Radiation-induced side effects are directly related to the doses received by normal tissues, including the salivary glands, pharyngeal constrictor muscles, mandible, spinal cord, cochlea/vestibular apparatus, and brainstem [13]. The 
development of multi-leaf collimators greatly advanced the conformality of radiation doses. Composed of sliding metal leaflets, multi-leaf collimators modulate the intensity of a radiation beam, thereby allowing clinicians to use either static, discrete radiation fields (intensity-modulated photon (X-ray) radiotherapy (IMRT)) or dynamic, contiguous arcs (volumetric arc radiotherapy (VMAT)) to "sculpt" the dose around the areas of interest [14, 15].

The ability of IMRT and VMAT to sculpt the radiation beam and thereby reduce the volume of high-dose radiation delivered to normal, non-targeted tissues has reduced the occurrence of several radiation-related side effects, including xerostomia and osteoradionecrosis. A meta-analysis of HNC studies comparing 2-dimensional and 3-dimensional (2D and 3D) radiation treatment techniques to IMRT found a $25 \%$ improvement in grade $2-4$ xerostomia (hazard ratio $=0.76,95 \%$ CI $0.66-0.87, P<0.0001$ ) with IMRT [16]. When used with prophylactic dental care, IMRT and VMAT have also led to substantial decreases in the incidence of osteoradionecrosis, the risk of which in modern studies has been estimated at less than 5\% [17, 18]. Although dysphagia continues to be a common side effect of radiotherapy, the lower mean doses to the uninvolved pharyngeal constrictor muscles and larynx that are achieved with IMRT and VMAT techniques have improved both the incidence and severity of symptoms [19].

Despite their ability to deliver conformal, high-dose radiation to targeted regions of the head and neck, IMRT and VMAT conversely distribute lower-dose radiation to a greater volume of non-targeted structures [20]. This collateral dose can have adverse clinical consequences. Rosenthal et al. demonstrated that, in the treatment of OPC, radiation dose to normal tissues located along the IMRT beam path resulted in worsening of several side effects relative to 3D conformal radiotherapy (3D-CRT), including high-grade oral mucositis [21]. Oral mucositis is associated with significant pain, dysphagia, weight loss, feeding tube placement, and unplanned hospitalizations. Severe mucositis, particularly when it results in treatment interruptions, can adversely influence disease control [22].

\section{Radiotherapy dose de-escalation}

Given the side effects associated with EBRT, radiation dose de-escalation has been investigated as a way of maintaining rates of disease control for HPVassociated OPC while reducing treatment-related morbidity [23]. Chera et al. studied patients with T0-T3, NO-N2c, M0 disease (according to the 7th edition of the American Joint Committee on Cancer (AJCC) staging system) who were treated with reduced-dose definitive chemoradiotherapy consisting of $60 \mathrm{~Gy}$ with weekly cisplatin $\left(30 \mathrm{mg} / \mathrm{m}^{2}\right)$. Surgical evaluation performed at 7-14 weeks after treatment completion demonstrated pathologic complete response rates of $98 \%$ for the primary site and $84 \%$ for involved lymph nodes [24]. Because pathologic response is a surrogate for clinical outcomes, this study served as proof of principle that reduced-dose radiation as definitive therapy may achieve favorable rates of locoregional control.

Several studies have also investigated outcomes for patients with HPVassociated OPC treated with de-escalated radiation doses after surgery [2527]. The Eastern Cooperative Oncology Group (ECOG) and the American 
College of Radiology Imaging Network (ACRIN) E3311 trial enrolled patients who were to receive transoral resection of the primary disease and neck dissection of lymph nodes. Those patients found postoperatively to be at intermediate risk of disease recurrence because of close margins at the primary site, evidence of perineural invasion/lymphovascular invasion, metastatic involvement of 2 to 4 lymph nodes, or metastatic involvement of least 1 lymph node and no more than $1 \mathrm{~mm}$ of extranodal extension were randomly assigned to receive either $50 \mathrm{~Gy}$ or $60 \mathrm{~Gy}$ of postoperative radiation [28•]. At a median follow-up interval of 31.8 months, the progression-free survival rates were 95.0\% (90\% CI 91.4-98.6) for those given 50 Gy and 95.9\% (90\% CI 92.699.3) for those given $60 \mathrm{~Gy}$. Additional studies are needed, but the results of this randomized phase II trial support the use of lower radiation doses for postoperative therapy in at least some instances of HPV-associated OPC.

Although induction chemotherapy is not considered part of standard of care for HPV-associated OPC, several trials have been conducted to evaluate induction chemotherapy followed by reduced-dose radiotherapy for those showing clinical response to the induction therapy [29-32]. The ECOG-ACRIN E1308 trial reported outcomes for patients with resectable, stage III or IV, HPVassociated OPC (per AJCC 7th edition) who received 3 cycles of induction chemotherapy followed by chemoradiotherapy to $54 \mathrm{~Gy}$ for those with clinical complete response at the primary site of disease. Among complete responders, the 2-year progression-free and overall survival rates were $80 \%$ (95\% CI 65-89) and $94 \%$ (95\% CI 82-98), respectively, and the reduced-dose radiotherapy was associated with a lower rate of acute, grade 3 dysphagia [31].

Despite the encouraging results from dose de-escalation studies, HPVassociated OPC does not have a uniformly favorable prognosis [33]. In RTOG 0129, Ang et al. demonstrated that the number of pack-years of tobacco smoking, tumor category, and nodal category all affected overall survival rates after radiotherapy, with more than 10 pack-years, larger tumors, and advanced adenopathy portending worse prognosis [34]. Although a pathologic complete response rate of $98 \%$ at the primary site after reduced-dose, definitive, chemoradiotherapy to $60 \mathrm{~Gy}$ (as noted by Chera et al.) is acceptable, the pathologic complete response rate of $84 \%$ for involved lymph nodes suggests that further studies are needed on the clinical implications of different responses at primary and nodal sites after de-escalated doses. Also, the 2-year progression-free survival rate of $80 \%$ for complete responders in the ECOG-ACRIN E1308 trial and the relatively short median follow-up times of dose de-escalation studies in general leave doubt regarding long-term locoregional outcomes. Collectively, these findings suggest that caution should be applied before dose de-escalation strategies are widely adopted in clinical practice.

\section{Proton therapy}

Intensity-modulated proton therapy is an advanced form of EBRT that may afford patients with HPV-associated OPC both high rates of disease control and reduced treatment-related morbidity through treatment deintensification and elimination of unnecessary collateral radiation without dose de-escalation [35]. For all X-ray (photon)-based EBRT treatment techniques (including 2D, 3D-CRT, IMRT, and VMAT), radiation dose is 
delivered to normal tissues along the entire path of the beam, including entrance and exit doses. In contrast, proton therapy deposits radiation dose in accordance with the Bragg peak, resulting in a lower entrance dose, delivery of the maximum dose in the target volume of interest, and sharp dose fall-off thereafter [36]. Combining beams of various energies results in complete coverage of the target volume and almost no radiation dose delivered distally (Fig. 1). Treatments can be delivered by using either a passive scatter or an IMPT technique (also referred to as "pencil beam scanning"), with the latter achieving greater dose conformality [37].

\section{Biological enhancement of proton therapy for HPV-positive squamous cell carcinoma}

Proton radiation dose is defined in terms of relative biological effectiveness (RBE) or the ratio of X-rays to protons required to produce a defined biological endpoint [38]. Relative biological effectiveness takes into consideration radiation dose, radiation fractionation, tissue type, and the linear energy transfer

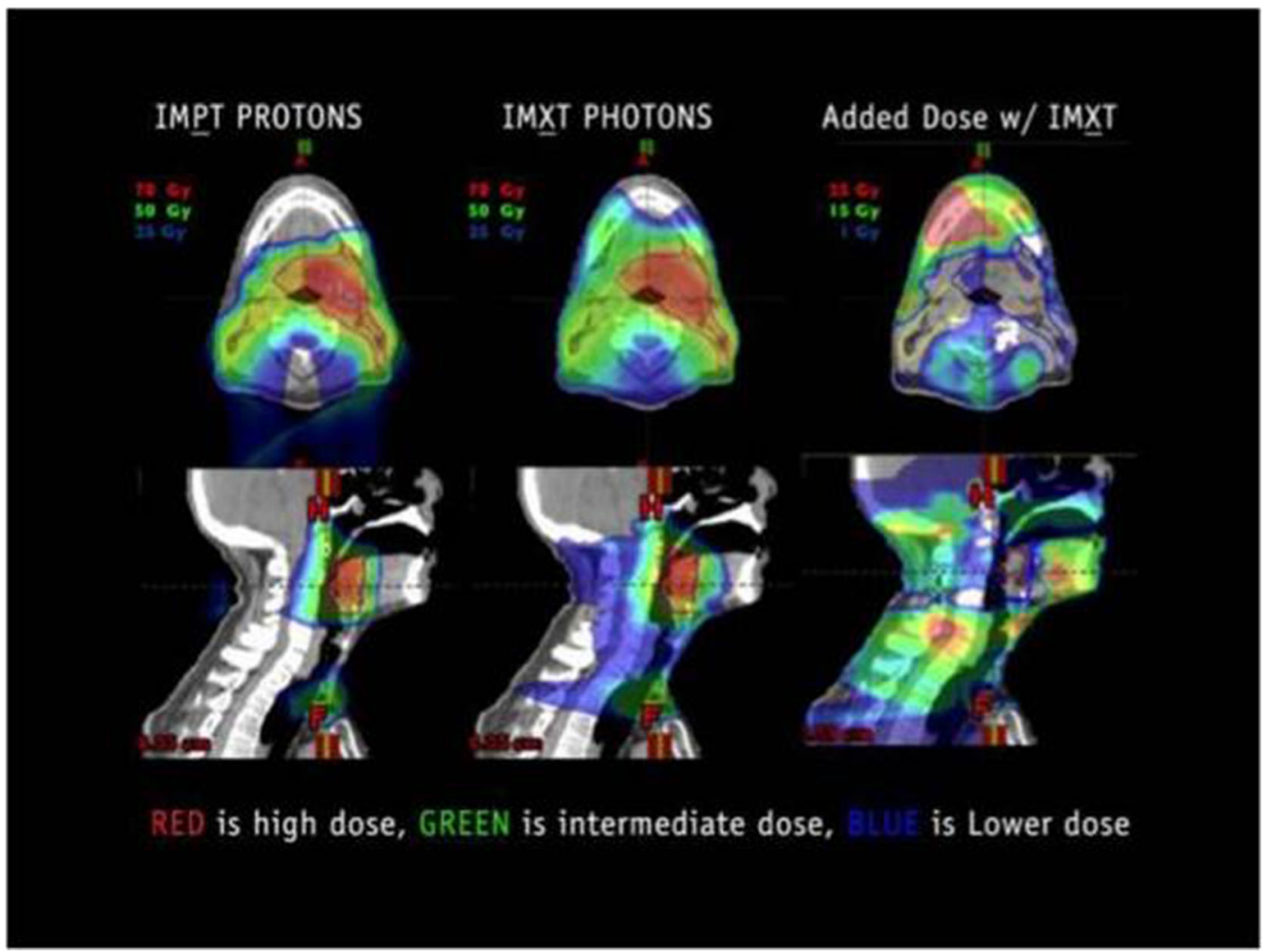

Fig. 1. Coronal (top) and sagittal (bottom) views of treatment plans used to assess dose distributions associated with intensitymodulated proton therapy (IMPT) (left) and intensity-modulated photon (X-ray) radiotherapy (IMXT) (middle). The images on the right illustrate the additional radiation dose associated with IMXT relative to IMPT. Reprinted from "Intensity Modulated Proton Therapy for Head and Neck Tumors: Gilding the Lily or Holy Grail?" by Steven J. Frank, International Journal of Radiation Oncology Biology Physics, volume 95, page no. 38, Copyright 2016, with permission from Elsevier. 
(LET) of protons in tissue. The RBE of protons is estimated to be 1.1, reflecting enhanced biological responses relative to X-rays of the same dose [38].

To investigate differences in cellular damage and mechanisms of cell death after proton versus photon radiation in the context of HPV-positive squamous cell carcinoma, Wang et al. delivered 4 Gy (RBE) of protons or 4 Gy of X-ray radiation to HPV-positive and HPV-negative squamous cell carcinoma cell lines $[39,40]$. Relative to the HPV-negative cell lines, clonogenic survival in the HPVpositive cell lines was lower at 10 and 17 days. When mechanisms of cell death were considered, proton radiation caused more mitotic catastrophe and larger percentages of senescent cells than did X-ray radiation, with the greatest increase in the percentage of senescent cells occurring in an HPV-positive cell line at 6 days [40].

Wang and colleagues subsequently investigated differences in protein expression after proton or photon therapy and the use of niraparib, a poly ADPribose polymerase (PARP)-1/2 inhibitor that acts to block deoxyribonucleic acid (DNA) damage repair, as a radiosensitizer [41, 42]. At $24 \mathrm{~h}$ after irradiation, higher levels of proteins associated with DNA damage repair were expressed in cells irradiated with protons, and these changes were more profound for HPVpositive cells. At a cell survival fraction of 0.1 , niraparib given with radiation increased the proton RBE by approximately $10 \%$ in HPV-positive cell lines compared with 3\% in HPV-negative cell lines [42]. Although additional studies are needed, these findings suggest that HPV-associated OPC may be more sensitive to proton radiation, and they form the foundation for future research regarding the delivery of targeted, systemic therapies with proton therapy to enhance its RBE.

\section{Proton therapy treatment planning}

Although a fixed RBE value of 1.1 for protons is used for radiation treatment planning, evidence exists to suggest that the RBE of protons varies along the path of the beam, being highest (and having highest LET) for the last few millimeters of the Bragg peak [43]. Given this source of variability in RBE, accurate estimation of the proton-beam range during treatment planning is crucial to ensure that the greatest dose is deposited within the target volumes. The range of a proton beam in a patient is a function of its initial energy and the ability of the patient's tissues to attenuate the proton, the so-called stopping power. The stopping power is estimated indirectly from the Hounsfield units (i.e., computed tomography (CT) numbers) of the various tissues through which the beam passes. Image distortion due to CT number uncertainty, as well as artifacts created by metal clips, dental fillings, and prostheses, can result in compromised stopping power calculations, inaccurate estimation of beam range, and deposition of high-dose radiation in normal structures [44].

In addition to accurate estimates of beam range, the clinical use of proton therapy requires careful consideration of uncertainties introduced by intratreatment weight loss, tumor shrinkage, and differences in patient positioning that can influence the size, shape, and location of target volumes and normal tissues during treatment [45]. In a retrospective analysis of 19 patients with OPC treated with IMRT, van Kranen et al. co-registered daily cone-beam CT scans obtained to verify patient positioning to treatment-planning CT scans and 
performed volumetric comparisons between structures of interest [46]. Although the cone-beam CT scans showed little change in the primary tumor volume between treatment fractions, a $4 \%$ per week reduction was noted in the volume of the parotid glands and a 10\% decrease was noted in the lymph node clinical target volumes by week 5 . Conversely, the pharyngeal constrictor muscles were found to increase in volume over the course of treatment. Because these changes have implications for radiation dosimetry-including coverage of target volumes, dose to normal tissues, radiation-related side effects, and locoregional disease control-the acquisition of verification CT scans with adaptive planning, as indicated, is necessary to ensure the fidelity of the treatment plans. Up to $40 \%$ of patients with $\mathrm{HNC}$ will require repeated planning as a result of anatomic changes noted on verification CT scans, and some patients will require several adaptive plans during the course of radiation treatment [47].

\section{Proton therapy for oropharyngeal cancer}

The first hospital-based, clinical proton therapy facility in the USA opened in 1990 at Loma Linda University Medical Center in California. Nearly a decade passed before the inauguration of the second facility at Massachusetts General Hospital [48]. Despite the initially slow uptake of proton therapy, the number of proton therapy centers has proliferated quickly in the USA over the past decade, with 15 centers in operation in 2014 increasing to 34 centers in operation and two additional centers under construction in 2020 [49]. In the following sections, we highlight the investigational experience with proton therapy for OPC, from in silico studies to ongoing randomized trials comparing IMRT with IMPT for OPC.

Cozzi et al. performed a dosimetric comparison of mixed photon-electron beam, 3D-CRT, IMRT, and proton plans for 5 HNC patients, including 3 cases of OPC, treated with 3D-CRT at the Oncology Institute of Southern Switzerland [50]. Except for the mixed photon-electron beam plan, all plans achieved comparable coverage of the target volume with $90 \%$ of the prescribed dose of $54 \mathrm{~Gy}(\mathrm{RBE})$. However, the proton plans were more favorable with respect to dose heterogeneity, maximum dose to the spinal cord, and dose received by two-thirds of the parotid glands (Table 1).

Similarly, Kandula et al. reported a dosimetric comparison of IMRT and IMPT plans for 5 patients who had been treated with 60-66 Gy of IMRT for HNC at MD Anderson, including 2 patients with OPC [51]. Although both IMRT and IMPT plans showed adequate coverage of target volumes, the IMPT plans resulted in reduced radiation dose to the oral cavity, salivary structures, and brainstem. A subsequent MD Anderson dosimetric comparison between IMRT and IMPT plans for 25 patients with OPC who had been treated with 6670 Gy (RBE) of IMPT reported lower mean doses to the anterior oral cavity (8.3 Gy (RBE) versus 31.0 Gy, $P<0.001$ ), posterior oral cavity (40.5 Gy (RBE) versus $54.3 \mathrm{~Gy}, P<0.001$ ), inferior pharyngeal constrictor muscle (32.8 Gy (RBE) versus $45.6 \mathrm{~Gy}, P<0.001$ ), and middle pharyngeal constrictor muscle (48.2 Gy (RBE) versus 57.0 Gy, $P=0.046$ ) with IMPT (Table 1) [52]. 


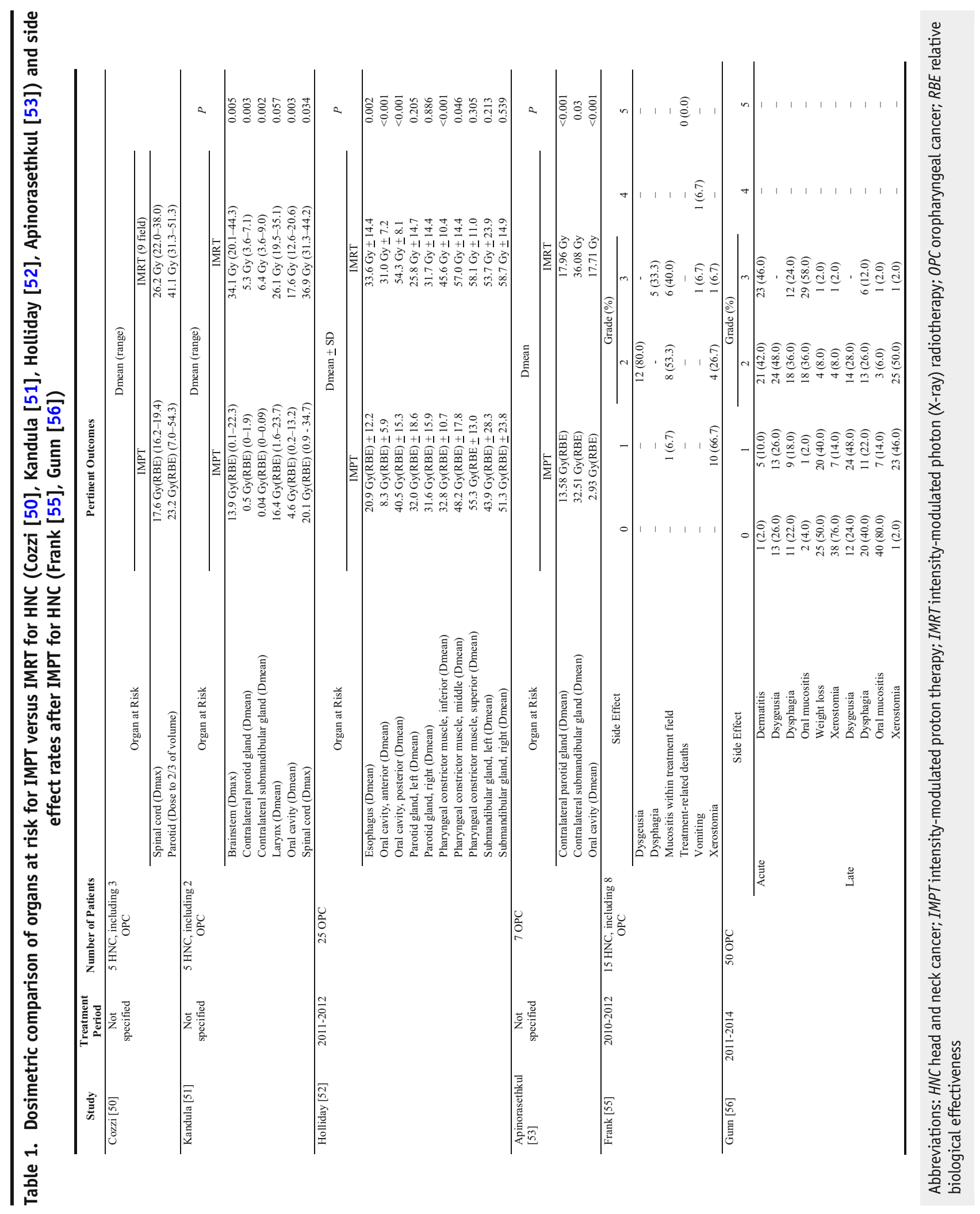


Apinorasethkul and colleagues compared treatment plans for IMRT versus IMPT for 7 patients with HPV-associated OPC who received IMPT postoperatively to doses of 60-63 Gy (RBE) [53]. All plans met planning parameters, including coverage of $95 \%$ of the target volume with the prescribed dose. However, proton plans were notable for lower mean doses to the oral cavity (2.93 Gy (RBE) versus $17.71 \mathrm{~Gy}, P<0.001$ ), contralateral parotid gland (13.58 Gy (RBE) versus $17.96 \mathrm{~Gy}, P<0.001$ ), and contralateral submandibular gland (32.51 Gy (RBE) versus $36.08 \mathrm{~Gy}, P=0.03$ ).

Loma Linda University Medical Center reported its early experience with proton therapy for 29 patients with stage III-IV OPC treated between October 1991 and June 2002 [54]. Patients were given 50.4 Gy of photon radiation followed by a concomitant boost of $25.5 \mathrm{~Gy}$ (RBE) delivered twice a day during the last 3.5 weeks of treatment, for a total tumor dose of 75.9 Gy. At a median follow-up time of 28 months (range 2-96 months), the 2-year rate of locoregional control was $93 \%$.

In 2013, Frank and colleagues reported the first clinical experience with the use of multifield-optimized IMPT for treating 15 patients with HNC, including 8 patients with OPC, at MD Anderson [55]. Patients received 66-70 Gy (RBE) of radiation (with or without concurrent chemotherapy) as definitive therapy, after surgery, or after induction chemotherapy. Regarding acute side effects, xerostomia was reported by all patients, but was severe (grade 3 ) in only 1 patient. Similarly, all patients experienced mucositis in the treatment field, including 6 patients with grade 3 symptoms (Table 1). However, no patients reported grade 2 or worse anterior oral mucositis. Two patients required placement of a feeding tube for grade 3 dysphagia, and $80 \%$ of patients reported grade 2 dysgeusia. At a median follow-up interval of 28 months (range 20-35 months), the overall clinical complete response rate was $93.3 \%$ (95\% CI 68.199.8).

Gunn and colleagues subsequently reported clinical outcomes for 50 patients with OPC treated with 66-70 Gy (RBE) of IMPT at MD Anderson from 2011 through 2014 [56]. Acute side effects included dermatitis, mucositis, xerostomia, and dysphagia. Acute grade 3 dysphagia occurred in a quarter of patients, and $20 \%$ of patients experienced inpatient hospitalization for poorly controlled pain, odynophagia, and dehydration secondary to oral mucositis. Twelve patients had feeding tubes placed ( 11 during treatment), and 5 of those patients required feeding tube nutrition for more than 3 months after completion of treatment. At a median follow-up time of 29 months (range 8-49 months), the 2-year overall survival rate was $94.5 \%$ (95\% CI 81.4-98.5) and the 2-year progression-free survival rate was $88.6 \%$ (95\% CI 75.8-95.1). An updated report of 103 patients with OPC treated from 2012 through 2016 showed that at a median follow-up time of 3.3 years (range $0.5-7.0$ years), the 3 -year overall survival, locoregional control, and disease-free survival rates were $96 \%, 93 \%$, and $93 \%$, respectively [57].

Aljabab et al. described the University of Washington Medical Center institutional experience with using IMPT to treat 46 patients with locally advanced OPC from March 2015 to August 2017 [58]. Patients received 70-74.4 Gy (RBE) definitively or 60-66 Gy (RBE) postoperatively. Acute grade 3 toxicities 
included dermatitis (76.1\%), mucositis (71.7\%), and xerostomia (6.5\%). One patient experienced weight loss of more than $10 \%$ from baseline and 2 patients were hospitalized for pain and dehydration. Feeding tubes were placed in 18 patients $(39.1 \%)$, with most $(n=14)$ inserted before the initiation of radiotherapy. No patient experienced late grade 3 or higher xerostomia or dysgeusia, and only 1 patient experienced late grade 3 or higher dysphagia (Table 2). At a median follow-up time of 19.2 months (interquartile range 11.2-28.4 months), rates of progression-free survival and overall survival were 93.5\% and 95.7\%, respectively.

In a case-control series, Blanchard and colleagues compared clinical outcomes of 150 patients with OPC treated at MD Anderson from 2010 through 2014, 50 with IMPT and 100 with IMRT [59]. Patients received 70 Gy concurrent with chemotherapy or 66 Gy definitively. Cases were matched in 1:2 fashion based on laterality of treatment, OPC subsite, HPV status, disease stage, receipt of concurrent chemotherapy, and smoking status. Analysis of side effects revealed less severe xerostomia at 3 months after IMPT versus IMRT (odds ratio $(\mathrm{OR})=0.38,95 \%$ CI $0.18-0.79$, $P=0.009$ ). At 12 months' follow-up time, rates of feeding tube placement or weight loss of more than $20 \%$ from pretreatment baseline also favored IMPT $(\mathrm{OR}=0.23,95 \% \mathrm{CI} 0.07-0.73, P=0.01)$. At a median follow-up time of 29 months (range 8-49 months), the 3-year locoregional control rate and the 3-year distant control rate with IMPT were $91.0 \%$ and $97.8 \%$, respectively. No significant difference was found in locoregional control or distant control between patients given IMPT versus those given IMRT.

In a study of osteoradionecrosis among 584 OPC patients treated with IMRT $(n=534)$ or IMPT $(n=50)$ at the same institution during an overlapping time interval, Zhang et al. found that, although no difference was observed between the two modalities with respect to the maximum dose to the mandible, the volume of the mandible receiving 5-70 Gy and the minimum, median, and mean doses were lower all with IMPT [60]. Forty-one patients treated with IMRT $(7.7 \%)$ and 1 patient treated with IMPT (2.0\%) developed osteoradionecrosis, with a median time to development of 11.4 months (range 6.7-16.1 months). All osteoradionecrosis developed in regions of the mandible that received at least $50 \mathrm{~Gy}$, and the volume of the mandible that received 45-70 Gy was significantly associated with osteoradionecrosis $(P<0.003)$.

Manzar et al. compared acute toxicities and patient-reported outcomes for 305 OPC patients treated with VMAT $(n=259)$ or IMPT $(n=46)$ at the Mayo Clinic from 2013 to 2018 with either 70 Gy definitively or 60-66 Gy postoperatively [61]. Analysis of feeding tube rates during and within 30 days of radiotherapy completion demonstrated lower use among patients treated with IMPT (19.6\% versus $46.3 \%$, OR $=0.27,95 \%$ CI $0.12-0.59, P=0.001)$. Patients treated with IMPT also experienced lower rates of acute hospitalization $(\mathrm{OR}=$ $0.21,95 \%$ CI $0.07-0.6, P=0.009)$. When the mean scores for measures of oral pain, mucositis, cough, sense of taste/smell, and use of nutritional supplements at end of treatment were compared with those at baseline, the differences in scores all favored IMPT (Table 2). At median follow-up times of 30 months for patients treated with VMAT and 12 months for those treated with IMPT, the 1- 


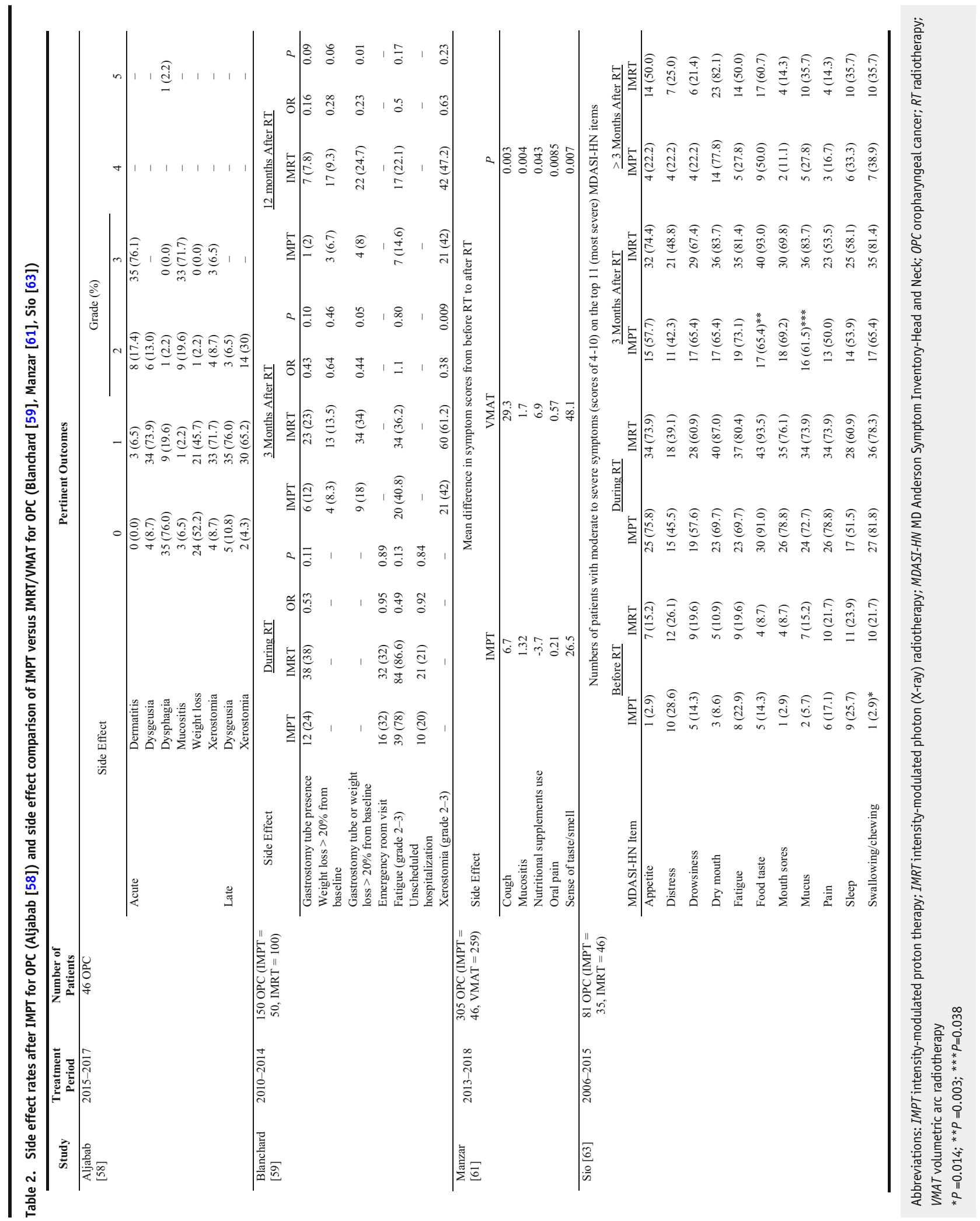


year rates of overall survival were $91.3 \%$ for VMAT and $92.6 \%$ for IMPT $(P=0.98)$.

The symptom burden associated with treatment, rated according to the MD Anderson Symptom Inventory for HNC (MDASI-HN), was reported for patients with OPC who received concurrent chemoradiation at MD Anderson from 2006 through 2015 with either IMRT $(n=46)$ or IMPT $(n=35)$ [62, 63]. Differences in proportions of patients experiencing decreased appetite favored IMPT at two intervals after treatment, first within 3 months of treatment completion (MDASI-HN average score 4.68 versus $6.37, P=0.048$ ) and later at more than 3 months after treatment completion (MDASI-HN average score 2.12 versus $4.14, P=0.036$ ). Symptom burden associated with changes in taste also favored IMPT within 3 months of treatment (MDASI-HN average score 5.76 versus $7.70, P=0.01$ ), but differences in this measure became nonsignificant during longer follow-up (Table 2). No significant difference was observed any of the other top 11 symptom scores (dry mouth, fatigue, pain, mucus, sleep, mouth sores, drowsiness, distress, swallowing) at baseline or follow-up. However, the average symptom burden for the top 5 symptom scores within 3 months of treatment completion favored IMPT (MDASI-HN average 5.15 versus $6.58, P=0.013$ ).

The "Randomized Trial of IMPT versus IMRT for the Treatment of Oropharyngeal Cancer of the Head and Neck" (NCT01893307) is the first prospective, phase II/III randomized trial to compare IMPT with IMRT for the treatment of

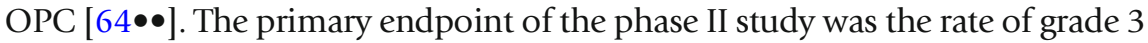
or higher treatment-associated side effects at 2 years. However, because these are physician-reported outcomes and side effects are often experienced differently by patients, this endpoint was deemed to lack objectivity and sensitivity. The primary endpoint was changed for the phase III trial to progression-free survival at 3 years, with secondary endpoints of physician-graded side effects and patient-reported outcome measures [55]. The non-inferiority, phase III design of this trial incorporates a measure of efficacy of IMPT and is consistent with the design of RTOG 1016 [10]. A cost-effectiveness analysis is also planned to clarify the overall value of proton therapy in light of its higher cost of delivery.

The "TOxicity Reduction using Proton bEam therapy for Oropharyngeal cancer (TORPEdO)" trial is the second, multicenter, phase III study of IMRT versus IMPT for OPC [65]. The primary endpoints of the trial are patientreported outcomes, as measured by the University of Washington physical toxicity composite score, and feeding tube dependence or severe weight loss at 12 months after treatment completion. Like the "Randomized Trial of IMPT versus IMRT for the Treatment of Oropharyngeal Cancer of the Head and Neck," the results of "TORPEdO" will include a cost-effective analysis. Enrollment at participating centers in the UK commenced in January 2020.

\section{Conclusions}

In contrast to the overall incidence of $\mathrm{HNC}$, the incidence of HPV-associated OPC has increased substantially over the past 2 decades. Its diagnosis in relatively young patients and its favorable prognosis have led to the 
development of treatment de-intensification strategies for HPV-associated OPC, including radiation dose de-escalation, in effort to reduce the likelihood of treatment-related side effects. However, because locoregional recurrence remains a source of treatment failure in HPV-associated OPC, the need persists for treatment techniques that permit treatment de-intensification to normal tissues without dose de-escalation to tumors. With appropriate patient selection, the physical and radiobiological properties of protons and the preclinical evidence demonstrating greater sensitivity of HPV-positive squamous cell carcinoma to proton radiation make proton therapy well suited to achieve this goal. The international experience with proton therapy suggests that, when compared with X-ray-based treatment, use of proton therapy for HPVassociated OPC confers comparable rates of disease control and reductions in both physician and patient-reported side effects. Given the inherent limitations of preclinical and retrospective studies of treatment for HPV-associated OPC, two trials currently underway, the "Randomized, Trial of IMPT versus IMRT for the Treatment of Oropharyngeal Cancer of the Head and Neck" and the "TOxicity Reduction using Proton bEam therapy for Oropharyngeal cancer (TORPEdO)," are expected to provide level 1 evidence regarding the indications for and value of proton therapy $[65,66]$.

\section{Declarations}

\section{Conflict of Interest}

Neil D. Gross was on the advisory board of PDS Biotechnology and Shattuck Labs and was a consultant for Intuitive Surgical. Renata Ferrarotto received personal fees from Regeneron-Sanofi, Ayala Pharmaceuticals, Prelude Pharmaceuticals, Bicara, Klus Pharma, Medscape, and Carevive; she declared institution conflicts of interest with Ayala Pharmaceuticals, AstraZeneca, Merck, Genentech, Pfizer, and Prelude Pharmaceuticals; and she was on the advisory boards of Ayala Pharmaceuticals, Prelude Pharmaceuticals, and Regeneron-Sanofi. Steven J. Frank received grants from C4 Imaging, Eli Lilly, and Hitachi; he received personal fees from Boston Scientific, C4 Imaging, and Varian; he was the founder/director of C4 Imaging; he was on the advisory board of Breakthrough Chronic Care and Varian; and he was a consultant for Boston Scientific and Varian. C. David Fuller received grants and personal fees from Elekta AB. Nicolette Taku received grants from Varian. Li Wang declares that she has no conflict of interest. Adam S. Garden declares that he has no conflict of interest. David I. Rosenthal declares that he has no conflict of interest. G. Brandon Gunn declares that he has no conflict of interest. William H. Morrison declares that he has no conflict of interest. Jack Phan declares that he has no conflict of interest. Jay P. Reddy declares that he has no conflict of interest. Amy C. Moreno declares that she has no conflict of interest. Michael Spiotto declares that he has no conflict of interest. Gregory Chronowski declares that he has no conflict of interest. Shalin J. Shah declares that he has no conflict of interest. Lauren L. Mayo declares that she has no conflict of interest. X. Ronald Zhu declares that he has no conflict of interest. Xiaodong Zhang declares that he has no conflict of interest.

\section{Human and Animal Rights and Informed Consent}

This article does not contain any studies with human or animal subjects performed by any of the authors. 


\section{Open Access}

This article is licensed under a Creative Commons Attribution 4.0 International License, which permits use, sharing, adaptation, distribution and reproduction in any medium or format, as long as you give appropriate credit to the original author(s) and the source, provide a link to the Creative Commons licence, and indicate if changes were made. The images or other third party material in this article are included in the article's Creative Commons licence, unless indicated otherwise in a credit line to the material. If material is not included in the article's Creative Commons licence and your intended use is not permitted by statutory regulation or exceeds the permitted use, you will need to obtain permission directly from the copyright holder. To view a copy of this licence, visit http://creativecommons.org/licenses/by/4.0/.

\section{References and Recommended Reading}

Papers of particular interest, published recently, have been highlighted as:

- Of importance

$\bullet \quad$ Of major importance

1. Siegel RL, Miller KD, Jemal A. Cancer statistics, 2020. CA Cancer J Clin. 2020;70(1):7-30.

2. Delaney G, Jacob S, Barton M. Estimation of an optimal external beam radiotherapy utilization rate for head and neck carcinoma. Cancer. 2005;103(11):2216-27.

3. Young D, Xiao CC, Murphy B, et al. Increase in head and neck cancer in younger patients due to human papillomavirus (HPV). Oral Oncol. 2015;51(8):72730.

4. Viens LJ, Henley SJ, Watson M, et al. Human papillomavirus-associated cancers-United States, 2008-2012. MMWR Morb Mortal Wkly Rep. 2016;65(26):661-6.

5. Mallen-St Clair J, Alani M, Wang MB, Srivatsan ES. Human papillomavirus in oropharyngeal cancer: The changing face of a disease. Biochim Biophys Acta. 2016;1866(2):141-50.

6. Adelstein DJ, Ridge JA, Brizel DM, et al. Transoral resection of pharyngeal cancer: summary of a National Cancer Institute Head and Neck Cancer Steering Committee Clinical Trials Planning Meeting, November 6-7, 2011, Arlington, Virginia. Head Neck. 2012;34(12):1681-703.

7. Nichols AC, Theurer J, Prisman E, et al. Radiotherapy versus transoral robotic surgery and neck dissection for oropharyngeal squamous cell carcinoma (ORATOR): an open-label, phase 2, randomised trial. Lancet Oncol. 2019;20(10):1349-59.

8. Deschuymer S, Mehanna H, Nuyts S. Toxicity reduction in the treatment of HPV positive oropharyngeal cancer: emerging combined modality approaches. Front Oncol. 2018;8:439.

9. Pfister DG, Spencer S, Adelstein D, et al. Head and neck cancers, version 2.2020, NCCN clinical practice guidelines in oncology. J Natl Compr Cancer Netw. 2020;18(7):873-98.
10. Gillison ML, Trotti AM, Harris J, et al. Radiotherapy plus cetuximab or cisplatin in human papillomaviruspositive oropharyngeal cancer (NRG Oncology RTOG 1016): a randomised, multicentre, non-inferiority trial. Lancet. 2019;393(10166):40-50.

11. Mehanna H, Robinson M, Hartley A, et al. Radiotherapy plus cisplatin or cetuximab in low-risk human papillomavirus-positive oropharyngeal cancer (De-ESCALaTE HPV): an open-label randomised controlled phase 3 trial. Lancet. 2019;393(10166):51-60.

12. Gebre-Medhin M, Brun E, Engström P, et al. ARTSCAN III: a randomized phase III study comparing chemoradiotherapy with cisplatin versus cetuximab in patients with locoregionally advanced head and neck squamous cell cancer. J Clin Oncol. 2021;39(1):38-47.

13. Dirix P, Nuyts S. Evidence-based organ-sparing radiotherapy in head and neck cancer. Lancet Oncol. 2010;11(1):85-91.

14. Bucci MK, Bevan A, Roach M 3rd. Advances in radiation therapy: conventional to $3 \mathrm{D}$, to IMRT, to $4 \mathrm{D}$, and beyond. CA Cancer J Clin. 2005;55(2):117-34.

15. Bedford JL, Warrington AP. Commissioning of volumetric modulated arc therapy (VMAT). Int J Radiat Oncol Biol Phys. 2009;73(2):537-45.

16. Marta GN, Silva V, de Andrade $\mathrm{CH}$, et al. Intensitymodulated radiation therapy for head and neck cancer: systematic review and meta-analysis. Radiother Oncol. 2014;110(1):9-15.

17. Ben-David MA, Diamante M, Radawski JD, et al. Lack of osteoradionecrosis of the mandible after intensitymodulated radiotherapy for head and neck cancer: likely contributions of both dental care and improved dose distributions. Int J Radiat Oncol Biol Phys. 2007;68(2):396-402.

18. Nabil S, Samman N. Risk factors for osteoradionecrosis after head and neck radiation: a systematic review. Oral Surg Oral Med Oral Pathol Oral Radiol. 2012;113(1):54-69. 
19. Wang X, Eisbruch A. IMRT for head and neck cancer: reducing xerostomia and dysphagia. J Radiat Res. 2016;57(Suppl 1):i69-75.

20. Mendenhall NP, Malyapa RS, Su Z, Yeung D, Mendenhall WM, Li Z. Proton therapy for head and neck cancer: rationale, potential indications, practical considerations, and current clinical evidence. Acta Oncol. 2011;50(6):763-71.

21. Rosenthal DI, Chambers MS, Fuller CD, et al. Beam path toxicities to non-target structures during intensitymodulated radiation therapy for head and neck cancer. Int J Radiat Oncol Biol Phys. 2008;72(3):747-55.

22. Vera-Llonch M, Oster G, Hagiwara M, Sonis S. Oral mucositis in patients undergoing radiation treatment for head and neck carcinoma. Cancer. 2006;106(2):329-36.

23. NRG Oncology. Reduced-dose intensity-modulated radiation therapy with or without cisplatin in treating patients with advanced oropharyngeal cancer. Available from: https://clinicaltrials.gov/show/ NCT02254278. Accessed September 1, 2020.

24. Chera BS, Amdur RJ, Tepper J, et al. Phase 2 trial of deintensified chemoradiation therapy for favorable-risk human papillomavirus-associated oropharyngeal squamous cell carcinoma. Int J Radiat Oncol Biol Phys. 2015;93(5):976-85.

25. Mayo Clinic. Evaluation of de-escalated adjuvant radiation therapy for human papillomavirus (HPV)-associated oropharynx cancer. Available from: https:// clinicaltrials.gov/show/NCT02908477. Accessed September 1, 2020.

26. Memorial Sloan Kettering Cancer Center. Major deescalation to $30 \mathrm{~Gy}$ for select human papillomavirus associated oropharyngeal carcinoma. Available from: https://clinicalTrials.gov/show/NCT03323463. Accessed September 1, 2020.

27. Eastern Cooperative Oncology Group. Transoral surgery followed by low-dose or standard-dose radiation therapy with or without chemotherapy in treating patients with HPV positive stage III-IVA oropharyngeal cancer. Available from: https://clinicaltrials.gov/show/ NCT01898494. Accessed September 1, 2020.

28. Ferris RL, Flamand Y, Weinstein GS, et al. Transoral robotic surgical resection followed by randomization to low- or standard-dose IMRT in resectable p16+ locally advanced oropharynx cancer: a trial of the ECOGACRIN Cancer Research Group (E3311). J Clin Oncol. 2020;38(15 suppl):650.

Randomized phase II trial of dose de-escalated, external beam radiotherapy given after surgery for patients with HPV-associated OPC.

29. Icahn School of Medicine at Mount Sinai. The Quarterback Trial: reduced dose radiotherapy for HPV+ oropharynx cancer. Available from: https:// clinicaltrials.gov/show/NCT01706939. Accessed September 1, 2020.

30. Chen AM, Felix C, Wang PC, et al. Reduced-dose radiotherapy for human papillomavirus-associated squamous-cell carcinoma of the oropharynx: a singlearm, phase 2 study. Lancet Oncol. 2017;18(6):803-11.

31. Marur S, Li S, Cmelak AJ, et al. E1308: phase II trial of induction chemotherapy followed by reduced-dose radiation and weekly cetuximab in patients with HPVassociated resectable squamous cell carcinoma of the oropharynx-ECOG-ACRIN Cancer Research Group. J Clin Oncol. 2017;35(5):490-7.

32. Seiwert TY, Foster CC, Blair EA, et al. OPTIMA: a phase II dose and volume de-escalation trial for human papillomavirus-positive oropharyngeal cancer. Ann Oncol. 2019;30(2):297-302.

33. Wirth LJ, Burtness B, Nathan CO, Grégoire V, Richmon J. Point/counterpoint: do we de-escalate treatment of HPV-associated oropharynx cancer now? And how? Am Soc Clin Oncol Educ Book. 2019;39:364-72.

34. Ang KK, Harris J, Wheeler R, et al. Human papillomavirus and survival of patients with oropharyngeal cancer. N Engl J Med. 2010;363(1):24-35.

35. Frank SJ. Intensity modulated proton therapy for head and neck tumors: gilding the lily or holy grail? Int J Radiat Oncol Biol Phys. 2016;95(1):37-9.

36. Smith AR. Proton therapy. Phys Med Biol. 2006;51(13):R491-504.

37. Moreno AC, Frank SJ, Garden AS, et al. Intensity modulated proton therapy (IMPT)-the future of IMRT for head and neck cancer. Oral Oncol. 2019;88:66-74.

38. Mohan R, Peeler CR, Guan F, Bronk L, Cao W, Grosshans DR. Radiobiological issues in proton therapy. Acta Oncol. 2017;56(11):1367-73.

39. Wang L, Wang X, Li Y, et al. Human papillomavirus status and the relative biological effectiveness of proton radiotherapy in head and neck cancer cells. Head Neck. 2017;39(4):708-15.

40. Wang L, Han S, Zhu J, et al. Proton versus photon radiation-induced cell death in head and neck cancer cells. Head Neck. 2019;41(1):46-55.

41. Wang L, Yang L, Han S, et al. Patterns of protein expression in human head and neck cancer cell lines differ after proton vs photon radiotherapy. Head Neck. 2020;42(2):289-301.

42. Wang L, Cao J, Wang X, et al. Proton and photon radiosensitization effects of niraparib, a PARP-1/-2 inhibitor, on human head and neck cancer cells. Head Neck. 2020;42(9):2244-56.

43. Frank SJ, Zhu R. J. Proton therapy: indications, techniques, and outcomes. 1st ed. Philadelphia: Elsevier; 2020. 272 p

44. De Marzi L, Lesven C, Ferrand R, Sage J, Boulé T, Mazal A. Calibration of CT Hounsfield units for proton therapy treatment planning: use of kilovoltage and megavoltage images and comparison of parameterized methods. Phys Med Biol. 2013;58(12):4255-76.

45. Bhide SA, Davies M, Burke K, et al. Weekly volume and dosimetric changes during chemoradiotherapy with intensity-modulated radiation therapy for head and neck cancer: a prospective observational study. Int J Radiat Oncol Biol Phys. 2010;76(5):1360-8. 
46. van Kranen S, Hamming-Vrieze O, Wolf A, Damen E, van Herk M, Sonke JJ. Head and neck margin reduction with adaptive radiation therapy: robustness of treatment plans against anatomy changes. Int J Radiat Oncol Biol Phys. 2016;96(3):653-60.

47. Yang Z, Zhang X, Wang X, et al. Multiple-CT optimization: an adaptive optimization method to account for anatomical changes in intensity-modulated proton therapy for head and neck cancers. Radiother Oncol. 2020;142:124-32.

48. Mohan R, Grosshans D. Proton therapy-present and future. Adv Drug Deliv Rev 2017;109:26-44.

49. The National Association for Proton Therapy. Proton therapy centers in the U.S. Available from: https:// www.proton-therapy.org/map/. Accessed on September 1, 2020.

50. Cozzi L, Fogliata A, Lomax A, Bolsi A. A treatment planning comparison of 3D conformal therapy, intensity modulated photon therapy and proton therapy for treatment of advanced head and neck tumours. Radiother Oncol. 2001;61(3):287-97.

51. Kandula S, Zhu X, Garden AS, et al. Spot-scanning beam proton therapy vs intensity-modulated radiation therapy for ipsilateral head and neck malignancies: a treatment planning comparison. Med Dosim. 2013;38(4):390-4.

52. Holliday EB, Kocak-Uzel E, Feng L, et al. Dosimetric advantages of intensity-modulated proton therapy for oropharyngeal cancer compared with intensitymodulated radiation: a case-matched control analysis. Med Dosim. 2016;41(3):189-94.

53. Apinorasethkul O, Kirk M, Teo K, et al. Pencil beam scanning proton therapy vs rotational arc radiation therapy: a treatment planning comparison for postoperative oropharyngeal cancer. Med Dosim.

2017;42(1):7-11.

54. Slater JD, Yonemoto LT, Mantik DW, et al. Proton radiation for treatment of cancer of the oropharynx: early experience at Loma Linda University Medical Center using a concomitant boost technique. Int J Radiat Oncol Biol Phys. 2005;62(2):494-500.

55. Frank SJ, Cox JD, Gillin M, et al. Multifield optimization intensity modulated proton therapy for head and neck tumors: a translation to practice. Int J Radiat Oncol Biol Phys. 2014;89(4):846-53.

56. Gunn GB, Blanchard P, Garden AS, et al. Clinical outcomes and patterns of disease recurrence after intensity modulated proton therapy for oropharyngeal squamous carcinoma. Int J Radiat Oncol Biol Phys. 2016;95(1):360-7.

57. Bahig H, Gunn GB, Garden AS, et al. Toxicity and pharyngeal dysphagia outcomes from intensity modulated proton therapy for oropharyngeal squamous cell cancer. Int J Radiat Oncol Biol Phys.

2019;105:E410.

58. Aljabab S, Liu A, Wong T, Liao JJ, Laramore GE, Parvathaneni U. Proton therapy for locally advanced oropharyngeal cancer: initial clinical experience at the University of Washington. Int J Part Ther. 2020;6(3):1-12.

59. Blanchard P, Garden AS, Gunn GB, et al. Intensitymodulated proton beam therapy (IMPT) versus intensity-modulated photon therapy (IMRT) for patients with oropharynx cancer-a case matched analysis. Radiother Oncol. 2016;120(1):48-55.

60. Zhang W, Zhang X, Yang P, et al. Intensity-modulated proton therapy and osteoradionecrosis in oropharyngeal cancer. Radiother Oncol. 2017;123(3):401-5.

61. Manzar GS, Lester SC, Routman DM, et al. Comparative analysis of acute toxicities and patient reported outcomes between intensity-modulated proton therapy (IMPT) and volumetric modulated arc therapy (VMAT) for the treatment of oropharyngeal cancer. Radiother Oncol. 2020;147:64-74.

62. Rosenthal DI, Mendoza TR, Chambers MS, et al. Measuring head and neck cancer symptom burden: the development and validation of the M. D. Anderson symptom inventory, head and neck module. Head Neck. 2007;29(10):923-31.

63. Sio TT, Lin HK, Shi Q, et al. Intensity modulated proton therapy versus intensity modulated photon radiation therapy for oropharyngeal cancer: first comparative results of patient-reported outcomes. Int J Radiat Oncol Biol Phys. 2016;95(4):1107-14.

$64 . \bullet$ Frank SJ, Blanchard P, Lee JJ, et al. Comparing intensitymodulated proton therapy with intensity-modulated photon therapy for oropharyngeal cancer: the journey from clinical trial concept to activation. Semin Radiat Oncol. 2018;28(2):108-1.

Summary of the design process of the first randomized trial to compare treatment with IMPT versus IMRT for patients with OPC.

65. Price J, Hall E, West C, Thomson D. TORPEdO-a phase III trial of intensity-modulated proton beam therapy versus intensity-modulated radiotherapy for multitoxicity reduction in oropharyngeal cancer. Clin Oncol (R Coll Radiol). 2020;32(2):84-8.

66. MD Anderson Cancer Center. Randomized trial of intensity-modulated proton beam therapy (IMPT) versus intensity-modulated photon therapy (IMRT) for the treatment of oropharyngeal cancer of the head and neck. Available from: https://clinicaltrials.gov/show/ NCT01893307. Accessed September 1, 2020

\section{Publisher's note}

Springer Nature remains neutral with regard to jurisdictional claims in published maps and institutional affiliations. 